\title{
MRI-based Synthetic CT in the Detection of Structural Lesions in Patients with Suspected Sacroiliitis: Comparison
} with MRI

\author{
Lennart B. O. Jans, MD, PhD* • Min Chen, MD* • Dirk Elewaut, MD, PhD • Filip Van den Bosch, MD, PhD • \\ Philippe Carron, $M D$, PhD ・Peggy Jacques, MD, PhD ・ Ruth Wittoek, MD, PhD ・ Jacob L. Jaremko, MD, PhD • \\ Nele Herregods, $M D, P h D$
}

From the Departments of Radiology (L.B.O.J., M.C., N.H.) and Rheumatology (D.E., F.V.d.B., P.C., P.J., R.W.), Ghent University Hospital, Corneel Heymanslaan 10, 9000 Ghent, Belgium; VIB Center for Inflammation Research, Unit for Molecular Immunology and Inflammation, Ghent University, Ghent, Belgium (D.E., F.v.d.B., P.C., P.J., R.W.); and Department of Radiology \& Diagnostic Imaging, University of Alberta Hospital, Edmonton, Canada (J.L.J.). Received April 11, 2020; revision requested May 11; revision received October 9; accepted October 30. Address correspondence to L.B.O.J. (e-mail: Lennart.jans@ugent.be).

*L.B.O.J. and M.C. contributed equally to this work.

Conflicts of interest are listed at the end of this article

See also the editorial by Fritz in this issue.

Radiology 2020; 00:1-7 • https://doi.org/10.1148/radiol.2020201537 • Content codes: MK MR CT

Background: Evaluation of structural lesions in the sacroiliac (SI) joints can improve the accuracy for diagnosis of spondyloarthritis. However, structural lesions, such as erosions, are difficult to assess on routine T1-weighted MRI scans.

Purpose: To determine the diagnostic performance of MRI-based synthetic CT (sCT) in the depiction of erosions, sclerosis, and ankylosis of the SI joints compared with T1-weighted MRI, with CT as the reference standard.

\begin{abstract}
Materials and Methods: A prospective study (clinical trial registration no. B670201837885) was performed from February 2019 to November 2019. Adults were referred from a tertiary hospital rheumatology outpatient clinic with clinical suspicion of inflammatory sacroiliitis. MRI and CT of the SI joints were performed on the same day. SCT images were generated from MRI scans using a commercially available deep learning-based image synthesis method. Two readers independently recorded if structural lesions (erosions, sclerosis, and ankylosis) were present on T1-weighted MRI, sCT, and CT scans in different reading sessions, with readers blinded to clinical information and other images. Diagnostic performance of sCT and T1-weighted MRI scans were analyzed using generalized estimating equation models, with consensus results of $\mathrm{CT}$ as the reference standard.
\end{abstract}

Resuls: Thirty participants were included ( 16 men, 14 women; mean age, 40 years \pm 10 [standard deviation]). Diagnostic accuracy of sCT was higher than that of T1-weighted MRI for erosion ( $94 \%$ vs $86 \%, P=.003)$, sclerosis $(97 \%$ vs $81 \%, P<.001)$, and ankylosis (92\% vs $84 \%, P=.04)$. With sCT, specificity for erosion detection $(96 \%$ [95\% CI: 90, 98] vs 89\% [95\% CI: 81, 94], $P$ $=.01]$ and sensitivity for detection of sclerosis [94\% [95\% CI: 87, 97] vs $20 \%$ [95\% CI: 10, 35], $P<.001]$ and ankylosis $(93 \%$ [95\% CI: 78, 98] vs 70\% [95\% CI: 47, 87], $P=.001$ ) were improved.

Conclusion: With CT as the reference standard, synthetic CT of the sacroiliac joints has better diagnostic performance in the detection of structural lesions in individuals suspected of having sacroiliitis compared with routine T1-weighted MRI.

( $\mathrm{RSNA}, 2020$

Online supplemental material is available for this article.

M RI of the sacroiliac (SI) joints is the imaging standard used to detect sacroiliitis in patients with spondyloarthritis (1). Bone marrow edema of the SI joints plays a key role in diagnosis but has limited sensitivity (65\%) and specificity $(75 \%)(1-3)$. Erosions of the SI joints are less prevalent in the setting of spondyloarthritis, with lower sensitivity (54\%) but much higher specificity (95\%) (2). T1-weighted MRI scans are obtained to depict structural lesions, such as erosions, which are notoriously difficult to demonstrate $(2,4)$.

CT also demonstrates erosions, as it enables clear visualization of bone due to its much higher x-ray attenuation. The combined use of MRI and CT is applied in radiation therapy planning (5) and orthopedic surgery $(6,7)$. However, performing both CT and MRI increases patient burden, adds ionizing radiation, and introduces complex workflows. It would be useful to generate synthetic CT (sCT) data demonstrating bone anatomy from MRI scans. Different techniques to generate sCT data were developed in the past decade, mainly for radiation therapy guidance $(8,9)$ and PET/MRI attenuation correction $(10,11)$.

In this study, we evaluate an sCT data generation method aiming at specific visualization of the osseous morphology by Hounsfield unit estimation (12). The MRI-based sCT is a deep learning-based technology, performing three-dimensional (3D) MRI to CT mapping and generating CT-like images from an axial 3D T1-weighted radiofrequency spoiled multiple gradient-echo (T1MGE) sequence. This technology was clinically validated in the cervical spine and pelvis (12-14). 


\section{Abbreviations}

sCT $=$ synthetic CT, $\mathrm{SI}=$ sacroiliac, $3 \mathrm{D}=$ three-dimensional, $\mathrm{T} 1 \mathrm{MGE}=$ T1-weighted radiofrequency spoiled multiple gradient echo

\section{Summary}

MRI-based synthetic CT improved specificity for erosion detection and sensitivity for sclerosis and ankylosis detection compared with T1-weighted MRI in individuals suspected of having sacroiliitis.

\section{Key Results}

- For individuals suspected of having sacroiliitis, MRI-based synthetic CT $(\mathrm{s} C \mathrm{~T})$ had greater diagnostic accuracy than T1-weighted MRI in the diagnosis of erosion ( $94 \%$ vs $86 \%, P=.003$ ), sclerosis (97\% vs $81 \%, P<.001)$, and ankylosis $(92 \%$ vs $84 \%, P=.04)$, with conventional CT as the reference standard.

- Interreader $\kappa$ values were $0.58-0.74$ for MRI, $0.70-0.90$ for $\mathrm{sCT}$, and $0.75-0.84$ for conventional CT.

The aim of this study was to determine the diagnostic performance of MRI-based sCT in the depiction of erosions, sclerosis, and ankylosis of the SI joints compared with T1-weighted MRI, with CT as the reference standard.

\section{Materials and Methods}

This prospective study (clinical trial registration no. B670201837885) was approved by the local ethics committee, and written informed consent was obtained from all participants. The authors had no conflicts of interest and had full control of inclusion of any data and information submitted for publication. None of the data from study participants has been reported previously.

\section{Study Participants}

From February 2019 to November 2019, individuals aged 18-60 years with inflammatory back pain for at least 3 months who were suspected of having inflammatory sacroiliitis by the rheumatologists at the outpatient clinic were referred. Exclusion criteria were pregnancy, metallic implants, contraindications to MRI, and failure to complete the imaging examination.

\section{MRI Protocol}

All MRI scans were obtained with a 3.0-T MRI unit (Prisma; Siemens Healthineers). A routine SI joint protocol was applied (parameters provided in Appendix E1 [online]). For sCT reconstruction, an additional axial 3D T1MGE sequence was performed (repetition time msec/echo time msec, 7/2, 3.5; field of view, $400 \times 400 \mathrm{~mm}$; acquisition matrix, $384 \times 384$; acquisition time, 5 minutes 37 seconds).

\section{Synthetic CT Reconstruction}

Synthetic CT images were reconstructed with commercially available software (BoneMRI Pelvic Region, version 1.1; MRIguidance). The software reconstructed sCT images from 3D T1MGE images using a deep learning method based on the U-net architecture (15). This method exploits local spatial contextual information in the multiecho data to reconstruct the latent bone structures, which was learned using paired MRI and CT data (12). The resulting sCT image expresses radiodensity contrast in Hounsfield units. The software runs on-site and is connected to the hospital picture archiving and communication system (PACS) via Digital Imaging and Communications in Medicine networking. The PACS automatically forwards the source MRI scans to the sCT software, which reconstructs sCT images and automatically returns them to the PACS in less than 30 minutes. No manual input is required. In this study, this was performed off-site at MRIguidance using a Horos PACS (version 3.3.5; Nimble).

\section{CT Protocol}

On the same day, participants underwent CT (Somatom Definition FLASH, Siemens Healthineers). Axial pelvic CT was performed using a dual-energy CT protocol (collimation, 32 $\times 0.6 \mathrm{~mm}$; pitch, 0.6 ; rotation time, 0.5 second; tube voltages, 140 and $100 \mathrm{kV}$ ). For further analysis, a weighted-average image was computed with a default ratio of 0.5:0.5 to resemble $120-\mathrm{kV}$ images.

\section{Image Analysis}

The sCT and CT images were reconstructed in a semicoronal direction (identical as T1-weighted; both section thickness and increment were $1 \mathrm{~mm}$ ). Two musculoskeletal radiologists (L.J., M.C.; 16 and 5 years of experience, respectively) independently scored the presence or absence of structural lesions in both SI joints. Each joint was divided into four quadrants for erosion and sclerosis scoring and into two halves (upper and lower) for ankylosis scoring. T1-weighted MRI, sCT, and CT scans were displayed and scored in different random order in three reading sessions, each separated by 4 weeks. Intrareader reliability was tested in a randomly selected subgroup of 15 participants by reader 1 with a 4-month interval. Both readers were blinded to all clinical data and other images. All images were evaluated using a Digital Imaging and Communications in Medicine viewer (RadiAnt, version 4.2.1; Medixant).

Structural lesions were defined as follows $(1,16,17)$ : An erosion on a T1-weighted scan is defined as a bony defect at the joint margin, with full-thickness loss of the dark signal of the subchondral cortex. An erosion on a CT or sCT scan is defined as a clear interruption of the subchondral cortical bone at the articular surface. Sclerosis is defined as subchondral low-intensity bands on T1-weighted scans with high attenuation values on CT or sCT scans extending at least $5 \mathrm{~mm}$ from the joint space. Ankylosis on T1-weighted, CT, and sCT images is defined as bone bridges across the joint with full-thickness loss of the subchondral bone plate on both sides of the joint. Only the cartilaginous compartment of the joint was scored. A consensus reading was performed for CT scores to generate a reference standard. Diagnostic confidence of every lesion score was evaluated using a four-point scale (18): 1 , poor confidence makes it almost impossible for diagnosis; 2 , low confidence that may affect the diagnosis; 3, moderate confidence that does not affect the diagnosis; and 4 , high confidence facilitating a clear diagnosis.

\section{Statistical Analyses}

The results of a prospective power analysis showed that a sample of 180 quadrants in 23 participants measured with both 
methods will achieve $80 \%$ power to detect an absolute improvement in accuracy of $10 \%$ with sCT, which is at the two-sided 5\% significance level using an exact conditional McNemar test, when we assumed the accuracy with conventional T1weighted MRI was $80 \%(19,20)$ and the correlation of accuracies between T1-weighted and sCT measurements was 0.19 (based on pilot data of 80 quadrants in 10 testing participants scanned before the start of the study). This calculation assumed no effect modification of the method used on the accuracy by the participant assessed (no participant $\times$ method interaction).

Intra- and interreader reliability were determined using the Cohen $\kappa$ statistic. As the scores from the same participant were not independent data, generalized estimating equation models for a binomial distribution with logit link were fitted to estimate the diagnostic performance and proportions of each diagnostic confidence level based on the scores from two readers. An exchangeable correlation structure was used to allow for a correlation between measurements from the same participant (across quadrants, readers, and methods). The fixed effects part of the model contained the method used. Estimated marginal probabilities and pairwise comparisons ( $\mathrm{sCT}$ vs T1-weighted MRI for diagnostic performance analysis; $\mathrm{SCT}$ vs T1-weighted MRI and sCT vs CT for diagnostic confidence analysis) were performed. All statistical analyses were performed using SPSS software (version 26.0; IBM). $P<.05$ was considered indicative of a significant difference.

\section{Results}

Participant inclusion is summarized in Figure 1. A summary of participant characteristics is shown in Table 1 . Thirty participants (16 men, 14 women) were included. The final clinical diagnosis for the 30 participants was axial spondyloarthritis $(n$ $=20)$; other arthropathy, degeneration, or anatomic variation $(n=6)$; and unknown diagnosis $(n=4)$. The mean age of the study participants was 40 years \pm 10 [standard deviation] (age range, $20-56$ years).

For consensus CT readings, erosion was present in 15\% (37 of 240) of quadrants in 33\% (10 of 30) of the participants, sclerosis was present in $24 \%$ (57 of 240) of quadrants in $60 \%$ of the participants (18 of 30), and ankylosis was present in $24 \%$ (29 of 120 ) of half joints in $27 \%$ (eight of 30 ) of the participants. The median number and interquartile range (IQR) of affected quadrants and half joints per participant is 0 (IQR, $0-1)$ for erosion, 1 (IQR, 0-4) for sclerosis, and 0 (IQR, 0-2) for ankylosis.

Intra- and Interreader Reliability

Intra- and interreader $\kappa$ values are displayed in Table E1 (online). The overall intrareader $\kappa$ values were $0.68-0.76$ on T1-weighted scans, $0.70-0.88$ on sCT scans, and $0.77-0.90$ on CT scans. Interreader $\kappa$ values were $0.58-0.74$ on T1weighted scans, $0.70-0.90$ on sCT scans, and $0.75-0.84$ on CT scans.
Table 1: Characteristics of the 30 Study Participants

\begin{tabular}{ll}
\hline Characteristic & Value \\
\hline Age (y) & $40 \pm 10(20-56)$ \\
Sex* & 16 \\
$\quad$ Male & 14 \\
$\quad$ Female & $1.7 \pm 0.4(1.2-2.6)$ \\
Time between MRI and CT $(\mathrm{h})^{\dagger}$ & $10.3 \pm 9.3(0.3-40)$ \\
Back pain duration (y) & \\
Clinical diagnosis & \\
$\quad$ Axial spondyloarthritis & $67(20 / 30)$ \\
$\quad$ Other arthropathy, degeneration, & $20(6 / 30)$ \\
$\quad$ or anatomic variation & $13(4 / 30)$ \\
$\quad$ Unknown diagnosis & $15(37 / 240)$ \\
Lesions on CT ${ }^{\ddagger}$ & $24(57 / 240)$ \\
$\quad$ Erosion & $24(29 / 120)$ \\
\hline Sclerosis & Ankylosis
\end{tabular}

Note.-Unless otherwise indicated, data are mean \pm standard deviation, with the range in parentheses.

* Data are numbers of participants.

${ }^{\dagger}$ One participant underwent CT 6 days after MRI without any treatment during the interval.

‡ Data are percentages, with the numerator and denominator in parentheses.

\section{Diagnostic Performance of $\mathrm{sCT}$ and T1-weighted MRI for Structural Lesions}

Examples of structural lesions on T1-weighted, sCT, and CT scans are displayed in Figures 2-5. Estimated diagnostic accuracy, sensitivity, and specificity of sCT and T1-weighted MRI are summarized in Table 2 . These estimated values were after adjustment for within-participant correlation effects across readers, quadrants, and methods. The results for each reader without statistical adjustments are displayed in Table E2 (on- 

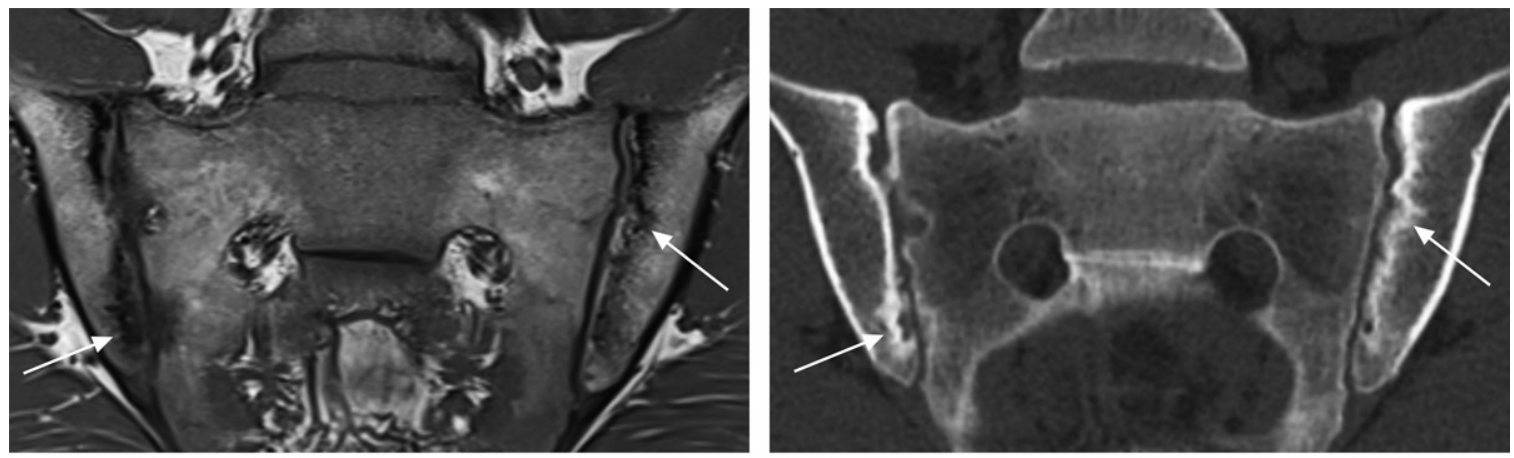

a.

b.

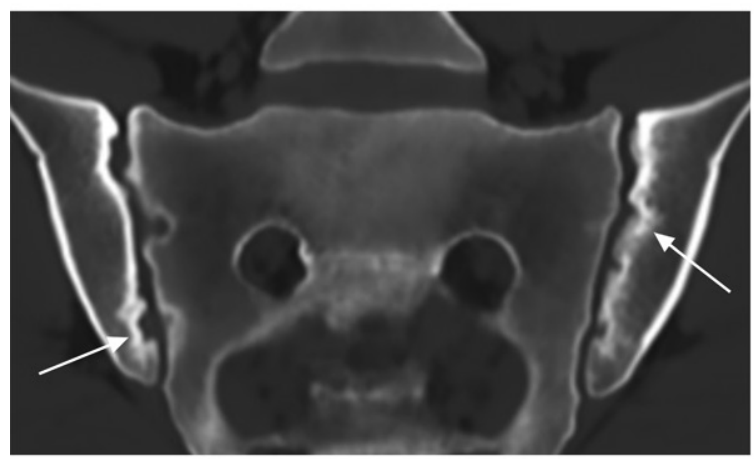

c.

Figure 2: Images in a 27-year-old man with sacroilititis. (a) TI-weighted MRI, (b) CT, and (c) synthetic CT scans. Erosions (arrows) are seen on both sacroiliac joints.
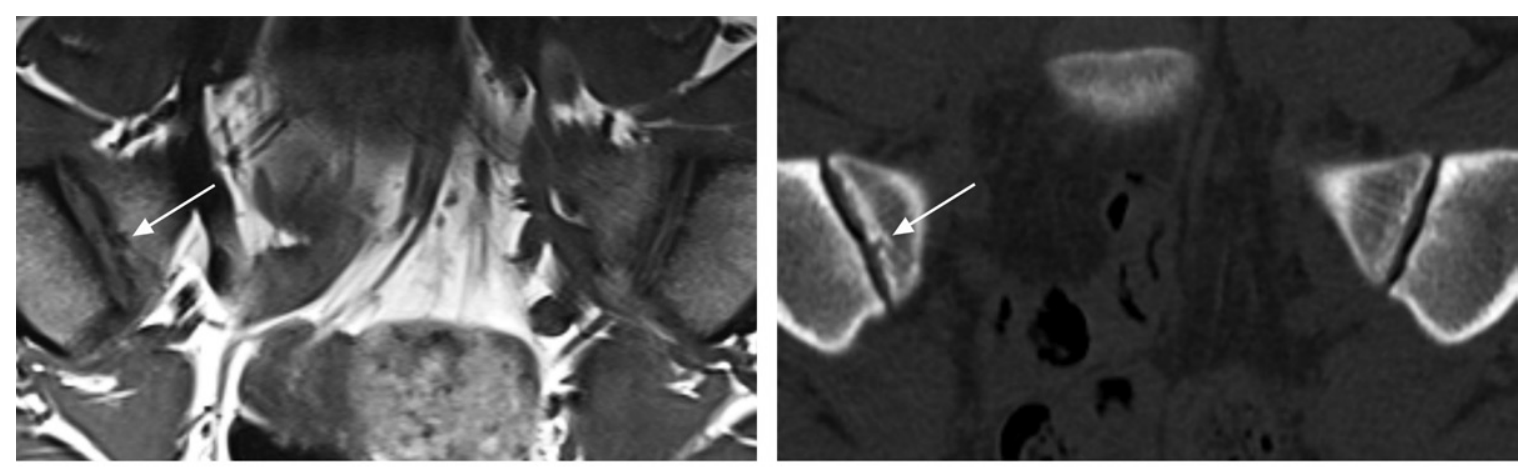

a.

b.

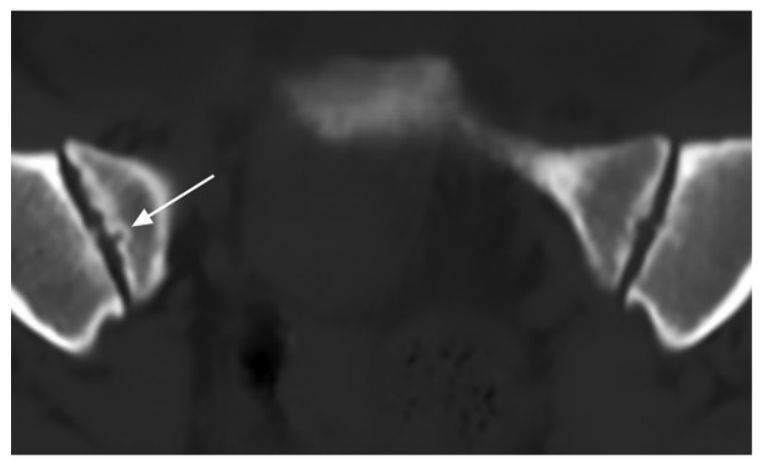

c.

Figure 3: Images in a 20-year-old woman with erosions. (a) T1-weighted MRI, (b) CT, and (c) synthetic CT scans. Small erosions (arrows) are clearly depicted on $\mathbf{c}$ but are less clear on $\mathbf{a}$. 


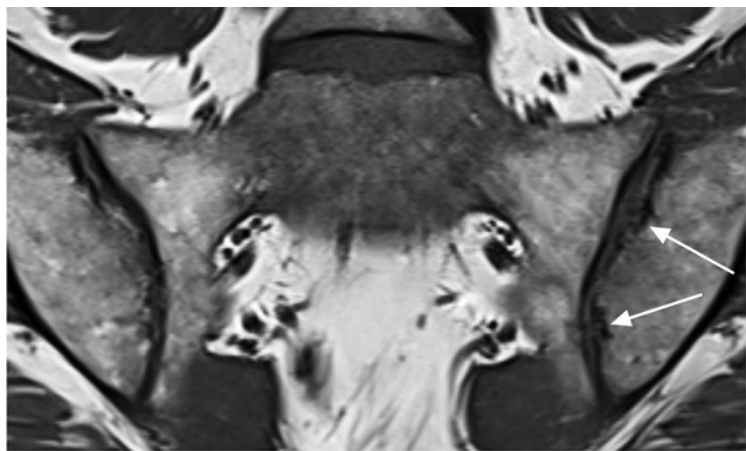

a.

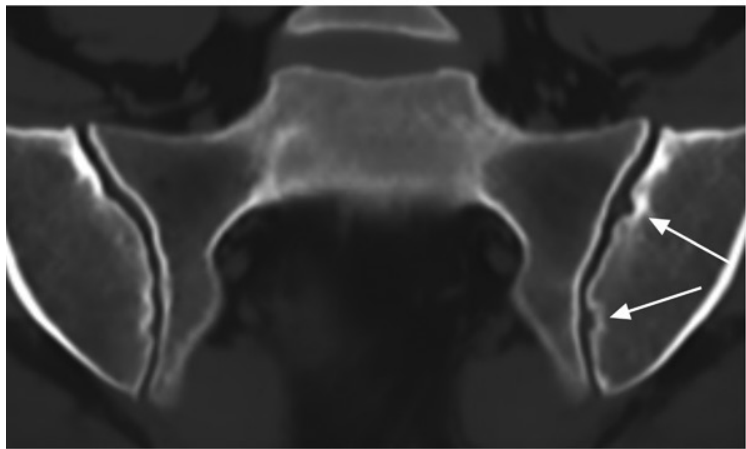

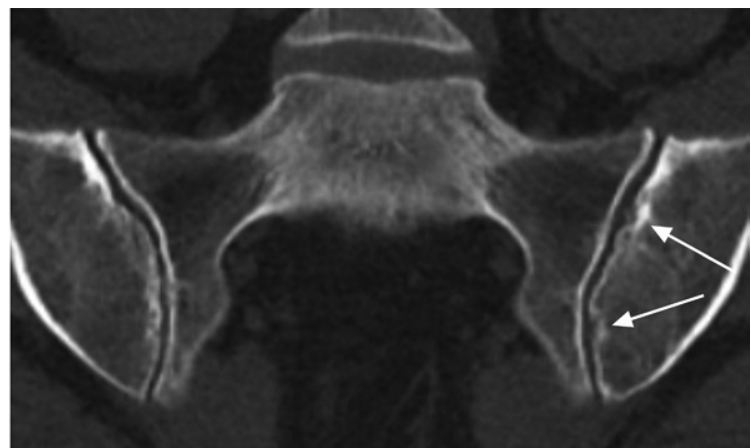

b.

c.

Figure 4: Images in a 51 -year old woman with erosions. (a) T1-weighted MRI, (b) CT, and (c) synthetic CT scans. Erosions (arrows) are clearly depicted on $\mathbf{c}$ but are less clear on $\mathbf{a}$

line). For erosion, the diagnostic accuracy and specificity of sCT were higher than those of T1-weighted MRI (accuracy: $94 \%$ [95\% CI: 88, 97] vs $86 \%$ [95\% CI: 79, 92], $P=.003$; specificity: 96\% [95\% CI: 90, 98] vs $89 \%$ [95\% CI: 81, 94], $P=.01)$. The diagnostic accuracy and sensitivity for sclerosis and ankylosis were higher for sCT than for T1-weighted MRI (sclerosis: accuracy, 97\% [95\% CI: 95, 98] vs $81 \%$ [95\% CI: 71, 88], $P<.001$; sensitivity, 94\% [95\% CI: $87 \%, 97 \%$ ] vs 20\% [95\% CI: 10\%, 35\%], $P<.001$ ); ankylosis: accuracy, $92 \%$ [95\% CI: $83 \%, 97 \%$ ] vs $84 \%$ [95\% CI: $71 \%, 92 \%$ ], $P=$ .04; sensitivity, 93\% [95\% CI: 78\%, 98\%] vs 70\% [95\% CI: $47 \%, 87 \%], P=.001$ ).

No differences were found in sensitivities for erosion or in specificities for sclerosis and ankylosis between sCT and T1weighted MRI. Results of diagnostic confidence scores are provided in Appendix E1 (online).

\section{Discussion}

This study prospectively compared the synthetic CT $(\mathrm{sCT})$ images generated with three-dimensional $\mathrm{T} 1$-weighted radiofrequency spoiled multiple gradient-echo sequence (T1MGE), conventional T1-weighted MRI, and CT in participants suspected of having sacroiliitis. Diagnostic accuracy in the detection of erosion ( $94 \%$ vs $86 \% ; P=.003)$, sclerosis $(97 \%$ vs $81 \%$; $P<.001)$, and ankylosis (92\% vs $84 \%, P=.04)$ was greater with sCT than with T1-weighted MRI. Inter- and intrareader reliability of $\mathrm{s} \mathrm{CT}$ were comparable to those of $\mathrm{CT}$ (intrareader $\kappa$ values, $0.70-0.88$ for $\mathrm{sCT}$ vs $0.77-0.90$ for $\mathrm{CT}$; interreader $\kappa$ values, $0.70-0.90$ for $\mathrm{s} C \mathrm{~T}$ vs $0.75-0.84$ for $\mathrm{CT}$ ).
Prior studies have assessed the use of MRI in the detection of osseous lesions. Diekhoff et al (21) and Baraliakos et al (19) applied 3D volumetric interpolated breath-hold examination (VIBE) gradient-echo sequences. They found that 3D VIBE depicted erosions in the SI joints with a higher sensitivity and without a loss of specificity compared with conventional T1weighted MRI. Recent studies explored generating radiographlike and CT-like images from MRI scans to improve osseous structure visualization. Gersing et al (22) found that MRI-derived radiograph-like images were feasible for bone tumor evaluation and were comparable to radiographs. Argentieri et al (23) and Breighner et al (24) presented MRI-based CT-like images based on zero echo time sequences and reported good agreement with CT of the spine and hips. However, this qualitative technique lacks specificity for cortical bone and requires specific hardware. In contrast, deep learning-based sCT was developed specifically to visualize osseous structures in a quantitative way based on attenuation maps; thus, it can accurately depict cortical and subcortical bone (12). Meanwhile, postprocessing of sCT images is a fully automatic process that does not require user input.

In our study, all structural lesions could be more reliably depicted with sCT than with T1-weighted MRI. The probability of having high diagnostic confidence with $\mathrm{sCT}$ was higher than with T1-weighted MRI in the detection of erosion and ankylosis. We found sCT significantly improved specificity in the detection of erosion without loss of sensitivity in comparison with T1-weighted MRI. This is useful because concerns have been raised about the specificity of MRI of SI joints in the diagnosis 


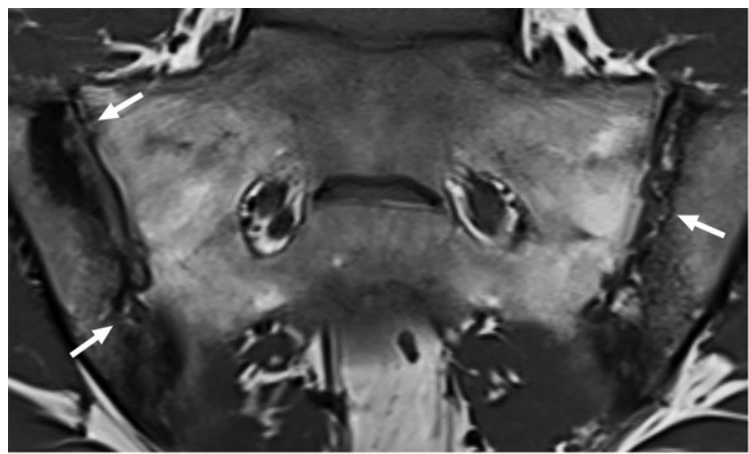

a.
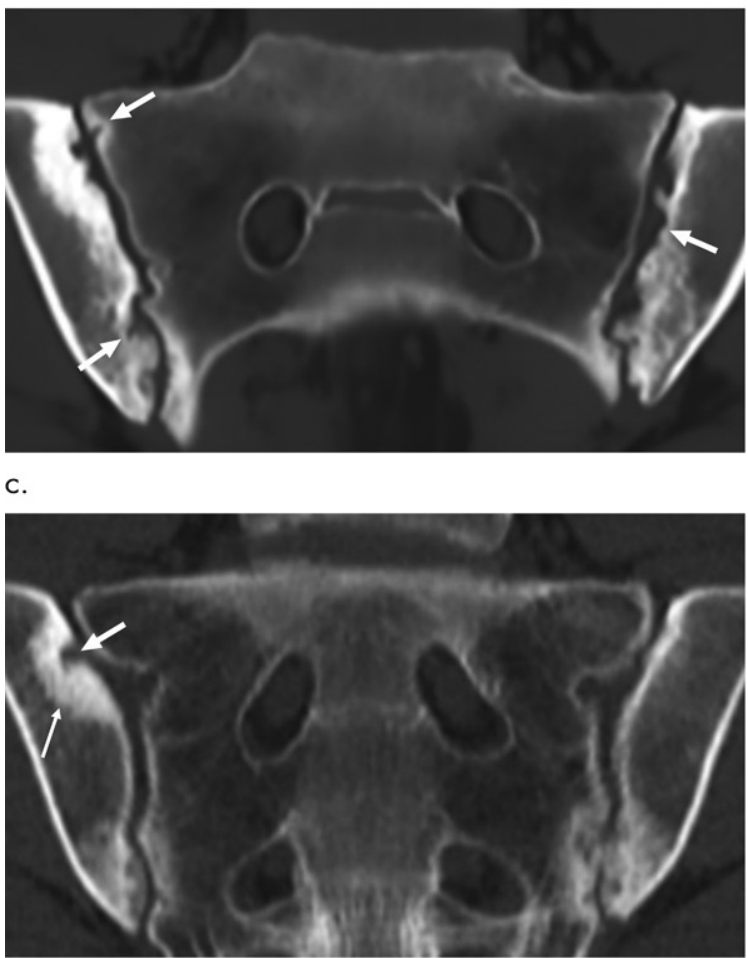

Figure 5: Images in a 28-year-old man with sacroilitis. (a, d) T 1 -weighted MRI, (b, e) CT, and $\mathbf{( c ,} \mathbf{f})$ synthetic CT scans. Extensive erosions in both sacroiliac joints (thick arrows) are better depicted on synthetic CT scans than on T1-weighted MRI scans and even more clearly than on CT scans. Subchondral sclerosis (thin arrow) is also better shown on synthetic CT scans than on T1-weighted MRI scans.

of spondyloarthritis (25). Also, sCT enabled us to more confidently visualize true erosions on MRI scans. For sclerosis and ankylosis, sCT improved sensitivity without a loss of specificity. As sclerosis and ankylosis are signs of bone repair after inflammation (17), this may also benefit the patients for follow-up and therapy guidance.

Our study had several limitations. Only a limited number of participants were included in the study. The diagnostic accuracy analysis was performed only on the quadrant level of the SI joint. Further studies with more participants are needed to compare sCT with T1-weighted MRI on the patient level or on the level of any other particular joint. All participants were referred from a tertiary hospital, which may represent a selection bias. No healthy control group was scanned for comparison. As diagnostic accuracy is dependent on disease prevalence, the estimated accuracies in our study are only valid in populations with the same prevalence.

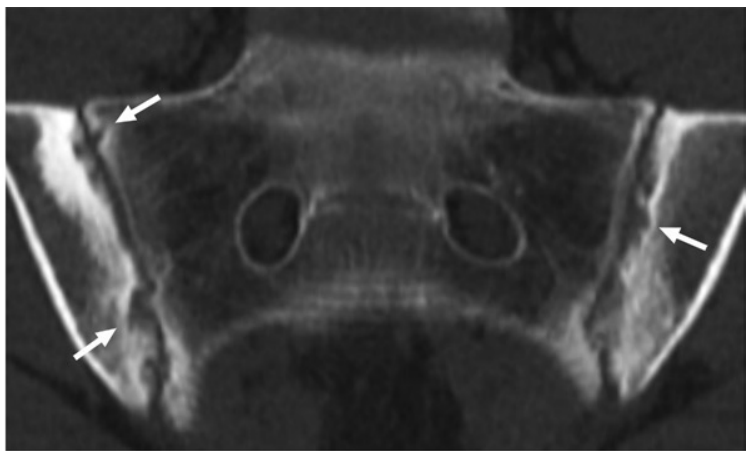

b.

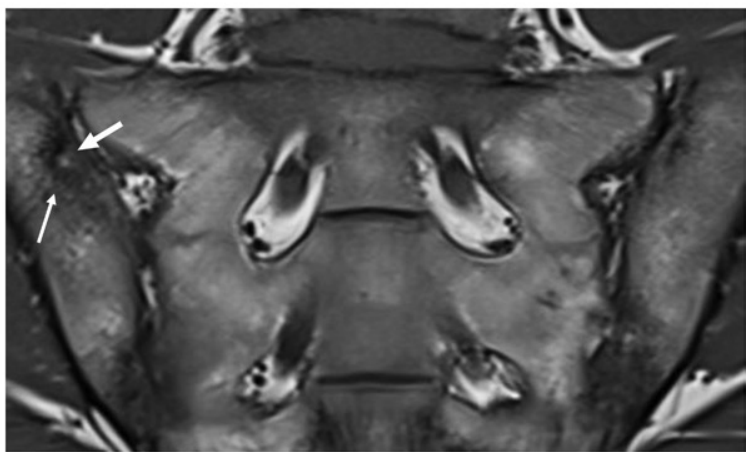

d.

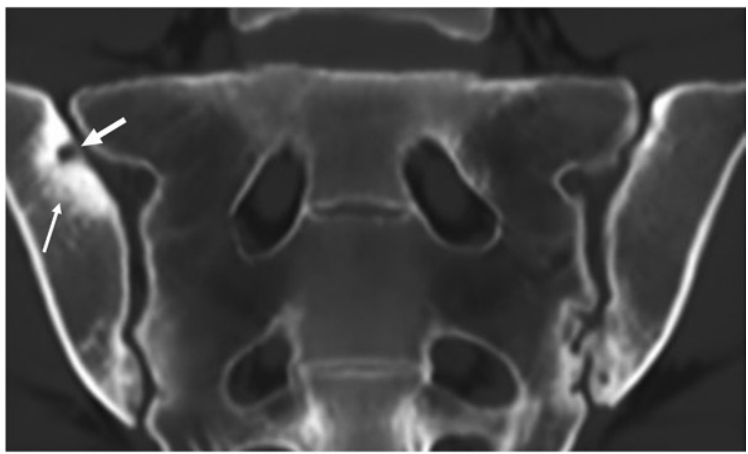

$f$
However, estimated specificities and sensitivities are independent from the disease prevalence. We limited the comparison of sCT to T1-weighted MRI, which is generally used for structural lesion detection in these patients. The diagnostic performance of the original 3D T1MGE sequence was not compared with sCT in the current study. The sCT images were reconstructed from two 3D T1MGE echo images, which contain unique but complementary information required to generate accurate $\mathrm{sCT}$ images with specific bone contrast. The added value of $\mathrm{s} C \mathrm{~T}$ over the original sequence for lesion detection was not studied and could be the goal of future studies. Finally, participants were scanned with a 3-T MRI unit, which limited the reported findings to one field strength.

In conclusion, we found that the synthetic CT scan reconstructed from an axial three-dimensional T1-weighted radiofrequency spoiled multiple gradient-echo sequence depicted 


\begin{tabular}{|c|c|c|c|}
\hline $\begin{array}{l}\text { Lesion and } \\
\text { Statistic }\end{array}$ & $\begin{array}{l}\text { Synthetic } \\
\text { CT }\end{array}$ & $\begin{array}{l}\text { T1-weighted } \\
\text { MRI }\end{array}$ & $P$ Value \\
\hline \multicolumn{4}{|l|}{ Erosion } \\
\hline Accuracy & $94(88,97)$ & $86(79,92)$ & .003 \\
\hline Sensitivity & $78(54,91)$ & $69(51,83)$ & .49 \\
\hline Specificity & $96(90,98)$ & $89(81,94)$ & .01 \\
\hline \multicolumn{4}{|l|}{ Sclerosis } \\
\hline Accuracy & $97(95,98)$ & $81(71,88)$ & $<.001$ \\
\hline Sensitivity & $94(87,97)$ & $20(10,35)$ & $<.001$ \\
\hline Specificity & $98(95,99)$ & $100(98,100)$ & .06 \\
\hline \multicolumn{4}{|l|}{ Ankylosis } \\
\hline Accuracy & $92(83,97)$ & $84(71,92)$ & .04 \\
\hline Sensitivity & $93(78,98)$ & $70(47,87)$ & .001 \\
\hline Specificity & $91(80,97)$ & $88(72,95)$ & .39 \\
\hline
\end{tabular}

Note.-Unless otherwise indicated, data are percentages, and data in parentheses are $95 \%$ CIs.

structural lesions of the sacroiliac joints with higher diagnostic accuracy and reliability than T1-weighted MRI, and with reliability comparable to that of CT.

Author contributions: Guarantor of integrity of entire study, L.B.O.J.; study concepts/study design or data acquisition or data analysis/interpretation, all authors; manuscript drafting or manuscript revision for important intellectual content, all authors; approval of final version of submitted manuscript, all authors; agrees to ensure any questions related to the work are appropriately resolved, all authors; literature research, L.B.O.J., M.C., F.V.d.B., P.C., N.H.; clinical studies, L.B.O.J., M.C., D.E., F.V.d.B., P.J., R.W.; statistical analysis, L.B.O.J., M.C.; and manuscript editing, all authors

Disclosures of Conflicts of Interest: L.B.O.J. disclosed no relevant relationships. M.C. disclosed no relevant relationships. D.E. disclosed no relevant relationships. F.V.d.B. disclosed no relevant relationships. P.C. disclosed no relevant relationships. P.J. disclosed no relevant relationships. R.W. Activities related to the present article: disclosed no relevant relationships. Activities not related to the present article: is a consultant for Union Chimique Belge, Abbvie, Tilman, Menarini, Merck Sharp and Dohme, and Galapagos. Other relationships: disclosed no relevant relationships. J.L.J. Activities related to the present article: disclosed no relevant relationships. Activities not related to the present article: disclosed no relevant relationships. Other relationships: is a cofounder of MEDO.ai, a start-up company developing artificial intelligence to automate US image analysis. N.H. disclosed no relevant relationships.

\section{References}

1. Sieper J, Rudwaleit M, Baraliakos X, et al. The Assessment of SpondyloArthritis international Society (ASAS) handbook: a guide to assess spondyloarthritis. Ann Rheum Dis 2009;68(Suppl 2):ii1-ii44.

2. Jans L, Coeman L, Van Praet L, et al. How sensitive and specific are MRI features of sacroiliitis for diagnosis of spondyloarthritis in patients with inflammatory back pain? JBR-BTR 2014;97(4):202-205.

3. Seven S, Østergaard M, Morsel-Carlsen L, et al. Magnetic Resonance Imaging of Lesions in the Sacroiliac Joints for Differentiation of Patients With Axial Spondyloarthritis From Control Subjects With or Without Pelvic or Buttock Pain:
A Prospective, Cross-Sectional Study of 204 Participants. Arthritis Rheumatol 2019;71(12):2034-2046.

4. Jans L, Egund N, Eshed I, Sudoł-Szopińska I, Jurik AG. Sacroiliitis in Axial Spondyloarthritis: Assessing Morphology and Activity. Semin Musculoskelet Radiol 2018;22(2):180-188.

5. Dirix P, Haustermans K, Vandecaveye V. The value of magnetic resonance imaging for radiotherapy planning. Semin Radiat Oncol 2014;24(3):151-159.

6. Wong KC. 3D-printed patient-specific applications in orthopedics. Orthop Res Rev 2016;8:57-66.

7. Wong KC, Kumta SM, Antonio GE, Tse LF. Image fusion for computer-assisted bone tumor surgery. Clin Orthop Relat Res 2008;466(10):2533-2541.

8. Edmund JM, Nyholm T. A review of substitute CT generation for MRI-only radiation therapy. Radiat Oncol 2017;12(1):28.

9. Kapanen M, Tenhunen M. T1/T2*-weighted MRI provides clinically relevant pseudo-CT density data for the pelvic bones in MRI-only based radiotherapy treatment planning. Acta Oncol 2013;52(3):612-618.

10. Leynes AP, Yang J, Shanbhag DD, et al. Hybrid ZTE/Dixon MR-based attenuation correction for quantitative uptake estimation of pelvic lesions in PET/MRI. Med Phys 2017;44(3):902-913.

11. Keereman V, Fierens Y, Broux T, De Deene Y, Lonneux M, Vandenberghe S. MRI based attenuation correction for PET/MRI using ultrashort echo time sequences. J Nucl Med 2010;51(5):812-818.

12. Florkow MC, Zijlstra F, Willemsen K, et al. Deep learning-based MR-to-CT synthesis: The influence of varying gradient echo-based MR images as input channels. Magn Reson Med 2020;83(4):1429-1441.

13. van der Kolk BY, van Stralen M, Podlogar M. Reconstruction of Osseous Structures in MRI scans of the Cervical Spine with BoneMRI: a Quantitative Analysis. ASNR 57th Annual Meeting, Boston, USA, May 18-23, 2019.

14. van Stralen M, van der Kolk BY, Zijlstra F, et al. BoneMRI of the cervical spine: Deep learning-based radiodensity contrast generation for selective visualization of osseous structures [abstr]. In: Proceedings of the Twenty-Seventh Meeting of the International Society for Magnetic Resonance in Medicine. Berkeley, Calif: International Society for Magnetic Resonance in Medicine, 2019.

15. Ronneberger O, Fischer P, Brox T. U-Net: Convolutional Networks for Biomedica Image Segmentation. In: Navab N, Hornegger J, Wells W, Frangi A, eds. Medical Image Computing and Computer-Assisted Intervention - MICCAI 2015. MICCAI 2015. Lecture Notes in Computer Science, vol 9351. Cham, Switzerland: Springer, 2015; 234-241.

16. Diekhoff T, Hermann KG, Greese J, et al. Comparison of MRI with radiography for detecting structural lesions of the sacroiliac joint using CT as standard of reference: results from the SIMACT study. Ann Rheum Dis 2017;76(9):1502-1508.

17. Maksymowych WP, Lambert RG, Østergaard M, et al. MRI lesions in the sacroiliac joints of patients with spondyloarthritis: an update of definitions and validation by the ASAS MRI working group. Ann Rheum Dis 2019;78(11):1550-1558.

18. Notohamiprodjo S, Stahl R, Braunagel M, et al. Diagnostic accuracy of contemporary multidetector computed tomography (MDCT) for the detection of lumbar disc herniation. Eur Radiol 2017;27(8):3443-3451.

19. Baraliakos X, Hoffmann F, Deng X, Wang YY, Huang F, Braun J. Detection of Erosions in Sacroiliac Joints of Patients with Axial Spondyloarthritis Using the Magnetic Resonance Imaging Volumetric Interpolated Breath-hold Examination. J Rheumatol 2019;46(11):1445-1449.

20. Xie R, Sun D, Morelli JN, Yin C, Xiong Y, Li X. Recognition of sacroiliac joint structural lesions: Comparison of volumetric interpolated breath-hold examination (VIBE) sequences with different slice thicknesses to T1-weighted turbo-echo. Eur J Radiol 2020;124:108849.

21. Diekhoff T, Greese J, Sieper J, Poddubnyy D, Hamm B, Hermann KA. Improved detection of erosions in the sacroiliac joints on MRI with volumetric interpolated breath-hold examination (VIBE): results from the SIMACT study. Ann Rheum Dis 2018:77(11):1585-1589.

22. Gersing AS, Pfeiffer D, Kopp FK, et al. Evaluation of MR-derived CT-like images and simulated radiographs compared to conventional radiography in patients with benign and malignant bone tumors. Eur Radiol 2019;29(1):13-21.

23. Argentieri EC, Koff MF, Breighner RE, Endo Y, Shah PH, Sneag DB. Diagnostic Accuracy of Zero-Echo Time MRI for the Evaluation of Cervical Neural Foraminal Stenosis. Spine 2018;43(13):928-933.

24. Breighner RE, Bogner EA, Lee SC, Koff MF, Potter HG. Evaluation of Osseous Morphology of the Hip Using Zero Echo Time Magnetic Resonance Imaging. Am J Sports Med 2019;47(14):3460-3468.

25. Poddubnyy D, Sieper J. Current Unmet Needs in Spondyloarthritis. Curr Rheumatol Rep 2019;21(9):43. 